\title{
The Compton trail of gamma-ray bursts: A long-after glow
}

\author{
D. Allard and E. Parizot
}

\author{
Institut de Physique Nucléaire d'Orsay, 15 rue Clémenceau, 91406 Orsay Cedex, France
}

Received 28 January 2004 / Accepted 16 March 2004

\begin{abstract}
As they travel through the gas of the host galaxy, some of the gamma-rays emitted in a Gamma-Ray Burst (GRB) may experience Compton scattering and reach an observer even if he is not located in the direction of the primary photon beam. Such a process will last until the GRB photons have left their host galaxy, and the ambient electron density becomes negligible. We investigate the observability of this indirect GRB light, which would be seen as a faint trail along the path of the GRB photons, long after the initial event. We find that the so-called Compton trail of a $10^{51}$ erg GRB can easily be observed from Earth, wherever the explosion occurred in our Galaxy in the past few thousand years. Gamma-ray surveys of the Galaxy can therefore provide constraints on the true GRB rate (or number of GRBs per supernova), independently of the GRB beaming angle. We also calculate the expected light curve and shape of the emitting region as a function of time.
\end{abstract}

Key words. gamma rays: bursts - gamma rays: theory - gamma rays: observations

\section{Introduction}

More than thirty years after the first observation of GammaRay Bursts (GRBs) in the late sixties (Klebesadel et al. 1973), their origin remains an open question. Remarkable progress has been made from both observational and theoretical points of view, essentially since the observations of the BATSE instrument on board CGRO (e.g. Fishman et al. 1994; Paciesas et al. 1999), and more significantly since the detection in 1997 of the first X-ray afterglow by the Beppo-SAX satellite (Costa et al. 1997). This allowed arc-minute localization, thanks to which optical afterglow could be detected, and in a number of cases a redshift could be determined, confirming the cosmological origin of GRBs. While it is now generally accepted that a highly relativistic fireball is involved (see the latest, extensive review by Zhang \& Mészáros 2004, and references therein), the GRB production mechanism(s) is (are) not known in detail. However, if one is only interested in the fate of the GRB photons after their emission, such details are unimportant and one can take advantage of the BATSE catalog, which contains about three thousand bursts and provides reliable information about the GRB phenomenology.

On a first approximation, one may simply regard a GRB as a huge number of gamma-ray photons between, say, $100 \mathrm{keV}$ and $1 \mathrm{MeV}$, emitted on a short time scale at a given place in a galaxy. As they propagate away from their source, these photons interact with the ambient interstellar medium (ISM) and in particular they are likely to be Compton scattered, either on the free electrons in the hot ISM or on the bound electrons in the atomic or molecular ISM. All along their path, some of the

Send offprint requests to: E. Parizot, e-mail: parizot@ipno.in2p3.fr primary photons will thus be scattered in all directions, so that a (not too distant) observer may be able to see the gamma-ray front propagating through the host galaxy, as a Compton trail lasting for a thousand years or more, depending on the thickness of the galaxy along the photon beam.

There is now strong evidence that GRBs have indeed a beamed emission (Frail et al. 2001), so that we can only observe the small fraction of them which are pointing towards us. But the beaming angle is not known in general, and there may be different types of GRBs in that respect. As a consequence, the true GRB rate in a given galaxy is quite uncertain, as there is a degeneracy in the rate/luminosity diagram: there could be either a few GRBs per Myr per Galaxy, with extremely high luminosity ( say $\sim 10^{53}$ erg or more), or much more frequent GRBs with correspondingly lower luminosity (and smaller beaming angle). While there has also been some evidence that (at least long-duration) GRBs are associated with massive stars, and maybe somehow related to type Ib/c supernovae, a natural question to ask is what fraction of these supernovae ( $\mathrm{SNe}$ ) may on average lead to, or be associated with a GRB. Estimates typically range from $\gtrsim 25 \%$ (Norris 2002) to only $\sim 1 \%$ of all type Ib/c SNe (Granot \& Loeb 2003), based on current estimates of GRB beaming angles (Frail et al. 2001). Given the estimated rate of these SNe, $\sim 2 \times 10^{-3} \mathrm{yr}^{-1}$ per $10^{10} L_{\odot}$ (Prantzos \& Boissier 2003), one can expect between 1 GRB every 2000 years and 1 GRB every 50000 years or so in our Galaxy.

Another estimate can be derived by correcting the BATSE rate by a beaming factor of $\sim 500$ (for the simplest uniform jet model; Frail et al. 2001) and by an additional factor of 2-3 if one includes X-ray flashes and X-ray rich GRBs. This would also lead to a rough event rate of 1 GRB every 2000 years per 
Galaxy, but the correction factors are quite uncertain. In particular, if the jets are not uniform but roughly Gaussian, the true GRB rate could be much lower (Zhang et al. 2004). Likewise, the evolution of the star forming rate should lead to a lower rate of local GRBs, compared to distant ones. The combination of both effects could result in a rate which is closer to the lower limit of the above estimate, or even below.

Any mechanism making it possible to infer the explosion of a GRB several thousand years in the past in nearby galaxies or even just in the Milky Way, would thus offer an opportunity to constrain the various models of GRBs, from the point of view of frequency. The above-mentioned Compton trail may be such a mechanism, as it allows one in principle to "detect" all GRBs, independently of their beaming angle and direction of emission. Indeed, the indirect glow made of Comptonscattered GRB photons would be visible from any direction (especially for photons with energy $E_{\gamma} \ll m_{\mathrm{e}} c^{2}=511 \mathrm{keV}$ ), so the Compton trail makes GRBs not only persistent, but also roughly isotropic sources.

In this paper, we investigate the shape and intensity of such Compton trails for various GRB parameters, and discuss the corresponding constraints on the true rate of GRBs inside a sphere determined by the instruments sensitivity.

\section{Compton scattering of GRB photons}

\subsection{Order of magnitude estimate}

Let us consider a GRB emitting photons of mean energy $E_{\gamma}=200 \mathrm{keV}$, with a total energy $E=E_{51} \times 10^{51} \mathrm{erg}$. This corresponds to a huge number of photons, of the order of $N_{\gamma} \sim 3 \times 10^{57} E_{51}$ ! Since $E_{\gamma}$ is much larger than the electron bounding energy in the interstellar gas, it makes no difference for the Compton effect whether the ambient electrons are bound or free, and since $E_{\gamma}<m_{\mathrm{e}} c^{2}=511 \mathrm{keV}$, we can approximate the Klein-Nishina cross section by the Thomson value, $\sigma_{\mathrm{T}} \simeq 665 \mathrm{mbarn}$. The Compton-scattered flux from a GRB exploding at a distance $D$ is thus approximately:

$\phi=\frac{N_{\gamma} n_{\mathrm{e}} \sigma_{\mathrm{T}} c}{4 \pi D^{2}} \approx\left(0.52 \mathrm{ph} \mathrm{cm}^{-2} \mathrm{~s}^{-1}\right) \times E_{51} n_{0} D_{\mathrm{kpc}}^{-2}$,

where $n_{\mathrm{e}}=n_{0} \times 1 \mathrm{~cm}^{-3}$ is the typical electron density in the ISM.

As can be seen, the fluxes obtained for Galactic GRBs are far above the detection thresholds of $\gamma$-ray satellites. It should also be noted that for $n_{\mathrm{e}}=1 \mathrm{~cm}^{-3}$, the Compton depth of the Galaxy is $\simeq 2 \times 10^{-3}$ per kpc, which means that the fraction of GRB photons which are Compton scattered is small, even if they propagate over several kiloparsecs along the Galactic plane. For this reason, we can neglect multiple scattering and consider that the primary $\gamma$-ray beam is not affected at all by the process.

Let us now turn to a more detailed calculation in order to find the time evolution of the fluxes, taking into account the geometry of the problem.

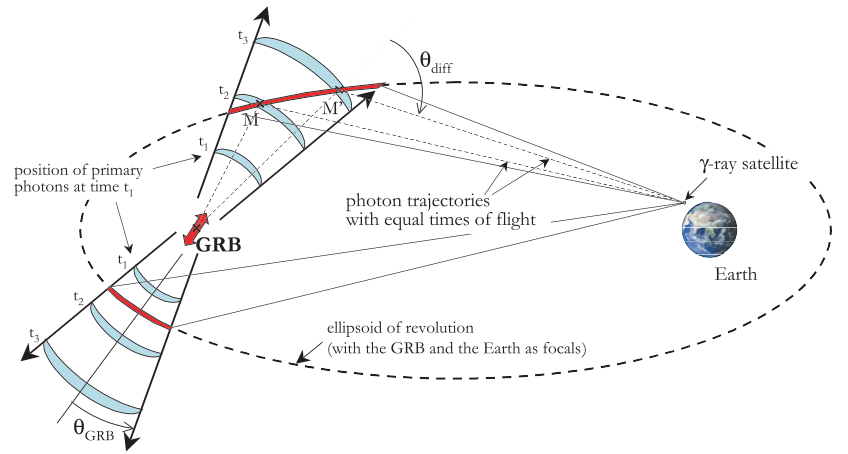

Fig. 1. A simplified scheme of the geometry of the problem. The emission region (intersection of the GRB cone and the ellipsoid, as discussed in the text) is shown in thick black line. The path of a photon scattered at point $\mathrm{M}$ towards the Earth (not to scale!) is also represented.

\subsection{The shape of the compton trail}

Let us consider a GRB occuring at a distance $D$ from Earth, which emits instantaneously $N_{\gamma} \gamma$-rays, uniformly distributed in a cone of opening angle $\theta_{\mathrm{GRB}}$. Once emitted, the photons scattered at any point $\mathrm{M}$ in the ISM can be observed at Earth if they have the appropriate scattering angle, $\theta_{\text {Diff }}$ (see Fig. 1). Obviously, the photons observed on Earth at a given time $t$ must have had the same time of flight, i.e. they have been scattered at points M such that "OM + MT = constant" (see Fig. 1). This defines an ellipsoid with foci O (the GRB central object) and $\mathrm{T}$ (the Earth). But of course the scattering centers must also lie within the GRB emission cone, so we can determine the set $\{\mathrm{M}\}$ of points involved in the indirect GRB emission detected at a given time as the intersection of the above-defined ellipsoid and the GRB emission cone (in practice, the ellipsoid has a thickness equal to $c \Delta t$, where $c$ is the speed of light and $\Delta t$ is the duration of the burst).

In the case where the Earth lies outside the GRB emission cone, the image of the corresponding Compton trail shows on the two-dimensional sky as an ellipse. This is a simple geometrical consequence of the fact that the primary emission point (i.e. the GRB central object) is a focus of the ellipsoid, and thus the contour of the intersection is a planar ellipse. If the emission is two-sided, of course, the full image actually consists of two ellipses, with relative intensities depending on their respective distance to Earth as well as on the local density of electrons in each region.

In the case where the Earth lies within the GRB emission cone (i.e. the direct light of the GRB could have been seen in the past), the Compton trail appears as a ring of gamma-ray light, with a radius increasing with time - or in fact as two rings with identical center (but one much smaller and fainter than the other) if the emission is two-sided. Note that the ring is not filled in that case, because the photons which were scattered at points lying inside the ring have already passed the Earth (since their way to the Earth was shorter). 


\subsection{Scattered gamma-ray fluxes}

Having identified the scattering region relevant to each observation time $t$, it is straightforward to calculate the Comptonscattered differential flux received in the detector: one simply needs to integrate over the appropriate volume the local scattering rate, attenuated according to the distance (see Appendix A for details on how to quickly perform the integration, taking advantage of the symmetries of the problem). The only subtlety lies in the fact that the photons observed at energy $E$ had a different energy, $E_{0}$, before the Compton interaction, which depends on the scattering angle, $\theta_{\text {diff }}$, according to:

$E_{0}=E\left[1-\frac{E}{m_{\mathrm{e}} c^{2}}\left(1-\cos \theta_{\mathrm{diff}}\right)\right]^{-1}$.

The resulting gamma-ray flux is then given by (see Appendix A):

$$
\begin{aligned}
\frac{\partial \phi(E, t)}{\partial E}= & \iiint_{\{M(t)\}} n_{\gamma}\left(t^{\prime}, E_{0}\left(E, \theta_{\mathrm{diff}}\right)\right) n_{\mathrm{e}}(M) \\
& \times \frac{\mathrm{d} \sigma_{\mathrm{C}}\left(E_{0}\left(E, \theta_{\mathrm{diff}}\right)\right)}{\mathrm{d} \omega} \frac{1}{M T^{2}} c \mathrm{~d} V,
\end{aligned}
$$

where $n_{\gamma}$ is the density of photons emitted at time $t^{\prime}=t-$ $(\mathrm{OM}+\mathrm{MT}) / c$, at energy $E_{0}$ (given by Eq. (2)), $n_{\mathrm{e}}(\mathrm{M})$ is the electron density at point $\mathrm{M}$, and $\mathrm{d} \sigma_{\mathrm{C}} / \mathrm{d} \omega$ is the differential Compton cross-section (the Klein-Nishina formula was used in the numerical calculation).

Assuming that the GRB emission is uniform over a time $\tau_{\mathrm{GRB}}$ and homogeneous inside a cone of opening angle $\theta_{\mathrm{GRB}}$, we have:

$n_{\gamma}\left(E_{0}, \mathrm{M}\right)=\frac{N_{\gamma}\left(E_{0}\right)}{2 \pi \mathrm{OM}^{2}\left(1-\cos \theta_{\mathrm{GRB}}\right) c \tau_{\mathrm{GRB}}}$.

As for the electron density field, since we are interested in the typical fluxes which can be expected for the Compton trail of a GRB in our Galaxy, we shall assume a smooth distribution of matter in the interstellar medium and use the model of Ferrière (1998), where the density is given as a function of galactocentric distance, $\rho$, and height $z$ above (or below) the Galactic disk, as a sum of exponential and Gaussian profiles (corresponding to molecular, cold neutral, warm neutral, warm ionized and hot gas).

\section{Results}

With the above prescriptions, the last ingredients which need to be specified in order to calculate the differential flux, Eq. (3), are the initial gamma-ray spectrum and of course the geometrical parameters of the GRB, namely its location, the orientation of the emission cone in the Galactic frame and its opening angle.

For the general purpose of this letter, it is appropriate to use a simple, generic GRB spectrum consisting asymptotically of two power-laws of indices $\alpha=-1.0$, and $\beta=-2.25$ (Band et al. 1993), with a peak in the energy distribution at $E_{\mathrm{p}}=$ $200 \mathrm{keV}: N_{\gamma}\left(E_{0}\right)=N_{0} B_{\alpha, \beta}\left(E_{0} / E_{\mathrm{p}}\right)$, where

$B_{\alpha, \beta}(x)= \begin{cases}x^{\alpha} \mathrm{e}^{-(2+\alpha) x} & \text { if } x \leq \frac{\alpha-\beta}{2+\alpha} \\ x^{\beta} \mathrm{e}^{\beta-\alpha}\left(\frac{\alpha-\beta}{2+\alpha}\right)^{\alpha-\beta} & \text { if } x \leq \frac{\alpha-\beta}{2+\alpha} .\end{cases}$

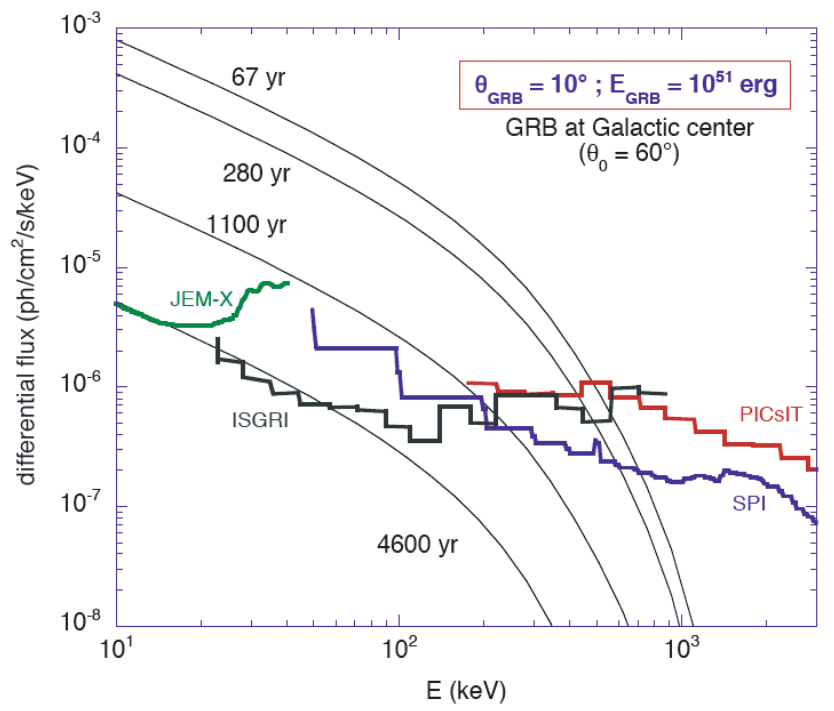

Fig. 2. Differential Compton-scattered flux received on Earth for a GRB of $10^{51}$ erg exploding at the Galactic center with the indicated parameters (see text). The fluxes are shown for four different times of observation after the explosion, and compared with the INTEGRAL thresholds.

Of course, a different primary energy spectrum would lead to a different scattered spectrum, and a structured jet would produce a non-uniform Compton trail, but the detection thresholds would remain roughly the same, as they depend mostly on global energetics.

Performing a numerical integration of Eq. (3), we have simulated GRB explosions with various geometrical parameters, at various places in the Galaxy. In Fig. 2, we show the differential flux of gamma-rays observed from the Compton trail of a GRB with an energy of $10^{51} \mathrm{erg}$ occurring at the Galactic center (GC) and emitting gamma-rays in a cone inclined at $60^{\circ}$ with respect to the rotation axis of the Galaxy in the plane perpendicular to the GC-Earth axis, with an opening angle of $10^{\circ}$. This may be considered as a typical GRB event in the Galaxy. Of course, closer (resp. more distant) GRBs will provide brighter (fainter) Compton trails. Likewise, if the angle between the emission axis and the GC-Earth axis is not $90^{\circ}$, then one of the two ellipses (or rings) will be brighter than the other (and also brighter than shown on the figure).

In Fig. 2, we have shown the differential spectrum of the Compton trail at various times after the explosion, together with the sensitivity of the four instruments onboard INTEGRAL. The integrated flux decreases as the primary photons move away from the Galactic center and pass through less and less dense material. For more (resp. less) inclined GRBs, the photons remain longer (shorter) in the dense parts of the interstellar medium, so that the Compton trails can be observed for a longer (shorter) time after the explosion. The value of $60^{\circ}$ is the median of the inclination distribution. As can be seen from Fig. 2, the Compton trail of a typical GRB at the Galactic center can be observed in principle up to a few thousand years after the explosion.

Another interesting thing to note from Fig. 2 is that the shape of the spectrum does not depend much on time. This 


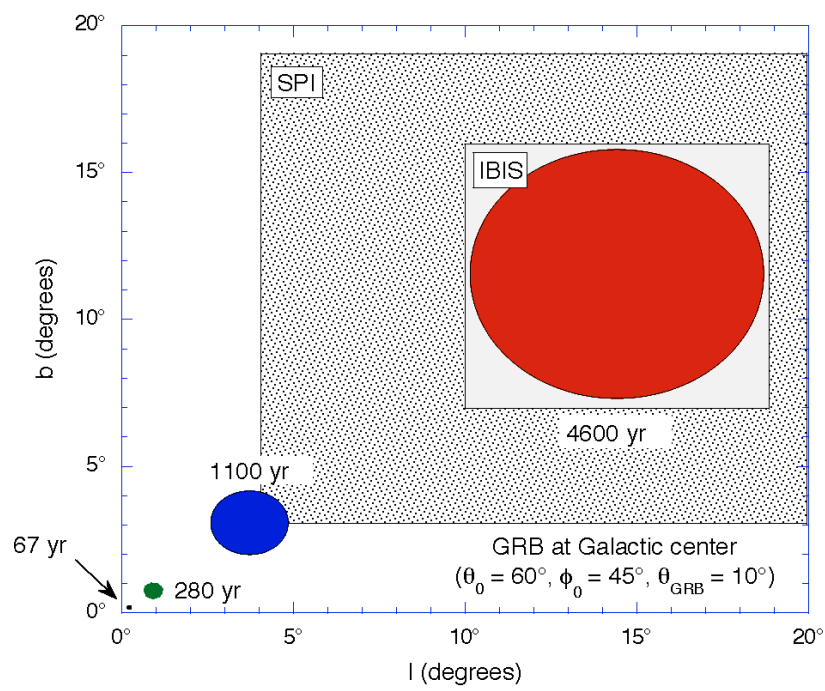

Fig. 3. Image of the scattering zone as seen from Earth for the same GRB parameters and the same times after explosion as in Fig. 2. The white and grey squares show the fields of view of IBIS and SPI, respectively.

is because the distribution of the Compton scattering angles (which determine the relation between the incoming and scattered photon energies) does not vary much for a given GRB geometry (unless the GRB is very close-by). As it turns out, only the high energy part of the spectrum is partially cut in the case of high scattering angles, i.e. when the scattering zone is far above the Galactic disk. As a consequence, one can derive a global detection threshold with INTEGRAL in terms of the integrated photon flux. For the typical spectral shape of a GRB Compton trail, we estimated that the overall threshold for a $3 \sigma$ detection with the INTEGRAL instruments is $\sim 1.1 \times 10^{-4} \mathrm{ph} / \mathrm{s} / \mathrm{cm}^{2}$ for an observation time of $10^{6} \mathrm{~s}$. We use this value as the limit for Compton trail detection. This is a conservative value, since the broad Galactic disk where most of the GRB Compton trails should lie if they exist will actually be scanned by INTEGRAL with a much longer exposure of up to several times $10^{7} \mathrm{~s}$, which will bring the sensitivity down to a significantly lower threshold.

The above sensitivity corresponds to a Compton trail fully contained in the instruments field of view (e.g. $9^{\circ} \times 9^{\circ}$ for IBIS and $16^{\circ} \times 16^{\circ}$ for SPI). This is the case for our typical GRB, as shown in Fig. 3 where the shapes and positions of the scattering region are indicated at the same four times as in Fig. 2. Closer trails would however be larger and could exceed the instruments field of view (although with a higher flux as well).

In Fig. 4, we show the integrated fluxes obtained for GRBs with $E=10^{51} \mathrm{erg}, \theta_{\mathrm{GRB}}=10^{\circ}$ and different distances and inclinations, compared to the estimated INTEGRAL sensitivity. The maximum time delay for the Compton trail detection is given for each case. One of the examples simulates what we could expect if the Crab supernova had been associated with a GRB. The observed peak in the scattered flux after $\sim 2000$ years is due to the photons crossing the denser Galactic disk (since the explosion occurred well above the plane). In all the cases

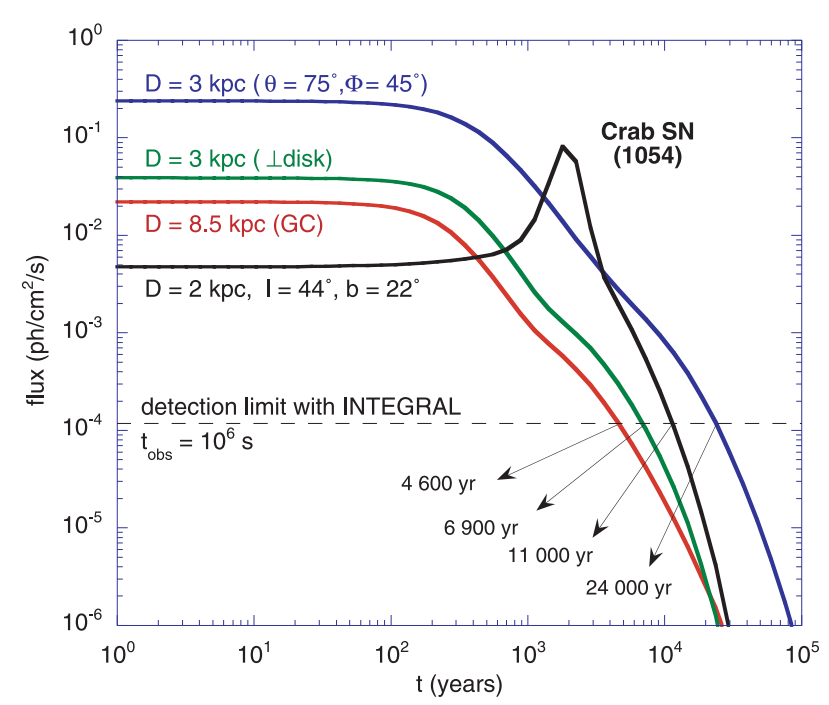

Fig. 4. Light curves of GRB Compton trails for various GRB parameters, as indicated. The "integrated INTEGRAL sensitivity" (see text) for an exposure of $10^{6} \mathrm{~s}$ is also shown, together with the time up to which the Compton trails would be detectable.

shown, the fluxes remain visible during thousands of years for a GRB of energy $10^{51} \mathrm{erg}$.

\section{Conclusion}

We have studied the possibility of observing the indirect light of GRBs resulting from the Compton scattering of the primary gamma-ray photons. We found that the Compton trail of a $10^{51}$ erg GRB could easily be observed from Earth, wherever the GRB exploded in our Galaxy in the last few thousands of years. The observability of lower (or higher) energy GRBs deduces straightforwardly from our results, by linear scaling. GRBs with energies as low as $10^{48}$ erg can thus be observed during a thousand years, provided they exploded within $\sim 4 \mathrm{kpc}$ from Earth. One should also note that GRBs with emission axes roughly parallel to the Galactic disk produce Compton trails which remain visible for a much longer time, up to $10^{5}$ years.

In addition to the potential astronomical interest of GRB Compton trails, the observation or non observation of such sources allows one, in principle, to constrain GRB models according to their total energy and (true) Galactic frequency. In particular, it is worth recalling that some models predict that a large fraction of all supernovæ actually produce GRBs, so that several GRBs would have exploded in our Galaxy in the last few thousands of years. Their Compton trail should thus be currently emitting its indirect light, and if no relevant signal were to be reported by current gamma-ray detectors, such models could be excluded. As recalled in the Introduction, more standard models predict an average time between GRB explosions between 2000 years and 50000 years. Thus, by searching for GRB Compton trails one should be able to explore interesting parts of the parameter space. It should be kept in mind, however, that lower rates are also possible, if the beaming effect is not so strong and the GRB rate is strongly reduced in evolved galaxies like ours. 
Although detecting an extended emission is generally not an easy task for gamma-ray instruments, we have shown that a typical GRB exploding around the Galactic center would fit entirely in the INTEGRAL field of view several thousand years after the explosion. It would also be very interesting to look for GRB Compton trails in other galaxies with future, more sensitive instruments. The order of magnitude calculation of Eq. (1) gives a good estimate of the required capabilities. An instrument with a continuum sensitivity below $5 \times 10^{-7} \mathrm{~cm}^{-2} \mathrm{~s}^{-1}$ would be able to see a GRB Compton trail up to $1 \mathrm{Mpc}$, for a $10^{51} \mathrm{erg}$ GRB, or even $10 \mathrm{Mpc}$ for a $10^{53} \mathrm{erg}$ GRB. Extending the volume of the universe where a GRB Compton trail could be observed would of course make the constraints on GRB frequencies (and models) more stringent.

It should also be emphasized that the detection of a Galactic Compton trail would provide interesting clues concerning the GRB progenitors. This is because the Compton trail geometry is well defined. In particular, one may be able to observe two elliptic sources (from the intersection of the ellipsoid with each half of the GRB emission cone), and geometrically identify the common origin of both trails, where the GRB remnant should lie.

Finally, we note that the luminosity of a Compton trail should not be expected to be uniform, even if the primary photons are uniformly distributed in angle in the GRB emission cone. The local emissivity is indeed related to the Comptonscattering rate, which is proportional to the ambient electron density. The intensity distribution in an angularly resolved Compton trail will thus reflect the matter distribution in the scattering region, and one may ideally think of the indirect GRB light as providing a scan of the interstellar medium along the path of the photons in the GRB emission cone, with slices just a few light-seconds thick.

Acknowledgements. The authors wish to thank Jacques Paul for useful discussions concerning the idea exposed in this paper and the capabilities of INTEGRAL, and Frédéric Daigne for sharing with us his knowledge of GRBs.

\section{Appendix A: Volume integration of the GRB Compton-scattered flux}

In this Appendix, we indicate how the integration of the Compton-scattered flux of the primary GRB gamma-rays can be performed in practice. In Sect. 2.2, we have shown that the instantaneous emission region (i.e. the region in space where the photons arriving simultaneously at time $t$ in the detector have been Compton-scattered) is the intersection of the GRB emission cone and a (thick) ellipsoid of revolution with the GRB explosion site and the detector as the two foci. In order to perform the integral in Eq. (3) over this region, one needs to choose a set of coordinates describing the volume and specify the limits of the single integrals involved in the calculation, or if one prefers the limiting coordinates which characterize the emission region explicitly.

It proves useful to use the symmetries of the problem and choose spherical coordinates in the frame where the origin is at the GRB explosion site, the $z$ axis (origin of the first angular coordinate, $\theta$ ) is the axis of the GRB emission cone, and the Earth is in the $x \mathrm{O} z$ plane, i.e. at $\phi=0$. Let $D$ be the distance between the Earth and the GRB. In the above frame, the Earth, $\mathrm{T}$, is represented by the spherical coordinates $\left(D, \theta_{\mathrm{T}}, 0\right)$, or by the cartesian coordinates $\left(D \sin \theta_{\mathrm{T}}, 0, D \cos \theta_{\mathrm{T}}\right)$.

The GRB emission cone is simply defined by:

$\theta \leq \theta_{\mathrm{GBR}}$

with arbitrary $r$ and $\phi$. As for the ellipsoid, we simply recall that the photons emitted at $\mathrm{O}$ and arriving at $\mathrm{T}$ after a time of flight $\tau=D / c+t$ are those which were scattered at points $\mathrm{M}$ of the ellipsoid $\mathcal{E}_{t}$ defined by $\mathrm{OM}+\mathrm{MT}=D+c t$, or MT $=c \tau+D-r$. Using the cartesian coordinates of $\mathrm{M}(r, \theta, \phi)=(r \sin \theta \cos \phi, r \sin \theta \sin \phi, r \cos \theta)$ to calculate MT, one gets:

$\mathcal{E}_{t}: \quad r=\frac{c t}{2} \times \frac{c t+2 D}{c t+A(\theta, \phi) D}$

where

$A(\theta, \phi) \equiv 1-\cos \theta_{\mathrm{T}} \cos \theta-\sin \theta_{\mathrm{T}} \sin \theta \cos \phi$.

If $\tau_{\mathrm{GRB}}$ is the duration of the GRB, the primary photons have been emitted from the source between $\tau=0$ and $\tau=\tau_{\mathrm{GRB}}$, or equivalently between $t=-D / c$ and $t=-D / c+\tau_{\mathrm{GRB}}$. The integration of the Compton-scattered flux received at time $t$, Eq. (3), must be done between ellipsoids $\mathcal{E}_{\left(t-\tau_{\mathrm{GRB}}\right)}$ and $\mathcal{E}_{t}$, since the contributing photons have had times of flight between $t+$ $D / c-t_{\mathrm{GRB}}$ and $t+D / c$.

The relevant points are thus easy to describe in our coordinate system. There is one of them for each couple $(\theta, \phi)$ where $\theta \leq \theta_{\mathrm{GRB}}$ and $-\pi \leq \phi \leq \pi$. Indeed, each half-line $(\theta, \phi)$ starts from $\mathrm{O}$, which is inside every ellipsoid $\mathcal{E}_{t}$, and goes to infinity. It thus crosses every ellipsoid $\mathcal{E}_{t}$ at point $\mathrm{M}(\mathcal{R}(\theta, \phi, t), \theta, \phi)$, where $\mathcal{R}(\theta, \phi, t)$ is simply given by Eq. (A.2).

Finally, the volume integration in Eq. (3) can be explicitly written as:

$\iiint_{\{M(t)\}} \mathrm{d} V \ldots=\int_{0}^{\theta_{\mathrm{GRB}}} \mathrm{d} \theta \int_{-\pi}^{\pi} \mathrm{d} \phi \int_{\mathcal{R}\left(\theta, \phi, t-\tau_{\mathrm{GRB}}\right)}^{\mathcal{R}(\theta, \phi, t)} r^{2} \sin \theta \mathrm{d} r \ldots$

\section{References}

Band, D., Matteson, J., Ford, L., et al. 1993, ApJ, 413, 281

Costa, E., Feroci, M., Frontera, F., et al. 1997, IAU Circ., 6572

Ferrière, K. 1998, ApJ, 503, 700

Fishman, G., Meegan, C., Wilson, R., et al. 1994, ApJ, 92, 229

Frail, D. A., Kulkarni, S. R., Sari, R., et al. 2001, ApJ, 562, 55

Granot, J., \& Loeb, A. 2003, ApJ, 593, L81

Klebesadel, R. W., Strong, I. B., \& Olson, A. O. 1973, ApJ, 182, 85

Norris, J. P. 2002, ApJ, 579, 386

Paciesas, W. S., Meegan, C. A., Pendleton, G. N., et al. 1999, ApJS, 122,465

Prantzos, N., \& Boissier, S. 2003, A\&A, 406, 259

Zhang, B., \& Mészáros, P. 2004, Int. J. Mod. Phys. A, in press [arXiv:astro-ph/0311321]

Zhang, B., Dai, X., Lloyd-Ronning, N. M., \& Mészáros, P. 2004, ApJ, 601, L119 